\title{
On the Role of Water in Heterogeneous Catalysis: A Tribute to Professor M. Wyn Roberts
}

\author{
Philip R. Davies ${ }^{1}$
}

Published online: 11 April 2016

(c) The Author(s) 2016. This article is published with open access at Springerlink.com

\begin{abstract}
From the earliest studies of heterogeneous catalysis, it was apparent that water plays a more important role in many systems than simply acting as a solvent. Its wide ranging effects have attracted increasing attention in recent years and was the topic of Prof. M.W. Roberts' final paper. The present review explores some of the latest work on water in reactions ranging from $\mathrm{CO}$ oxidation to Fischer-Tropsch catalysis, the different mechanisms proposed for its role are discussed and compared.
\end{abstract}

Keywords Water - Transient · Hydroxide - Catalysis . Heterogeneous catalysis · Gold

\section{Introduction}

Roberts studied many aspects of surface science and catalysis during his productive career, from the surface chemistry of wool [1] to the reactivity of carbon dioxide [2]. Amongst many notable contributions to the subject a thought provoking history of catalysis [3] is also worth mentioning, but in his last paper [4] Roberts focused his attention on the topic of water in catalysis which, as he pointed out, has been the subject of considerable debate from as far back as 1922 when Boswell showed that catalytic hydrogenation over nickel [5] and platinum [6] required its presence. Boswell's conclusion was reinforced by Beeck in 1935 when he showed [7] that the

Philip R. Davies

daviespr@cf.ac.uk

1 Cardiff Catalysis Institute, School of Chemistry, Cardiff University, Cardiff CF10 3AT, UK dehydrogenation of alkanes, alkenes and alcohols over platinum and carbon coated platinum filaments also required the presence of water. In his paper, Beeck pointed out the difficulties of determining water's precise role in these reactions and the fact that after 80 years of study, the different roles played by water in reactions at interfaces remains a "hot" topic attests to the accuracy of his insight.

In the following discussion we consider a selection of recent studies which illustrate the importance of water in heterogeneous catalysis and the different ways in which it can be involved. We are concerned primarily with reactions and so will not discuss in any detail the extensive literature detailing the structure of water films $[8,9]$ nor the role of hydroxyls in the nucleation and stabilisation of nanoparticles at oxide surface, an area that we have discussed else where [10] and which has recently been reviewed by Sterrer and Freund [11]. We will also not examine the surface science of water which was reviewed comprehensively by Thiel and Madey [12] and updated by Henderson [13]. The aim instead, is to explore some of the ideas contained in Roberts' final work and put them into the broader context of recent advances in the surface reactivity of water.

\section{Experimental and Theoretical Studies of Water in Heterogeneous Catalysis}

\subsection{The Influence of Water on Carbon Monoxide Oxidation over Gold Surfaces}

The important role of water in the oxidation of carbon monoxide over gold/titania catalysts was reported in the earliest papers on the catalyst and investigated more systematically in 2001 [14]. Increasing the water 
concentration to $\sim 200 \mathrm{ppm}$ increases the reaction rate by a factor of up to $\times 10$ the rate in the absence of water, Fig. 1, but leads to poisoning of the reaction rate at higher concentrations probably because of site blocking. Haruta concluded that water adsorbed on the catalyst was more important than water in the gas phase [14] echoing Beeck's conclusions from 70 years earlier on water participation in the hydrogenation reactions at platinum surfaces [7]. In a related paper with Cunningham [15], Haruta suggests the involvement of $\mathrm{OH}$ in the oxidation reaction at the interface between $\mathrm{MgO}$ and the small gold clusters and Bond and Thompson have also speculated that the mechanism involves $\mathrm{OH}$ at the edges of gold clusters [16]. Direct evidence for the reaction of $\mathrm{CO}$ with hydroxyls on a gold surface comes from a molecular beam study using oxygen isotope labelling to track the reactive species activating $\mathrm{CO}$ on $\mathrm{Au}(111)$ [17]. The isotope studies showed $\mathrm{CO}_{2}$ formation from both the chemisorbed "atomic" oxygen and hydroxyls formed from the reaction of the oxygen with labelled water. However, the study does not rule out the possibility that the $\mathrm{CO}$ is exclusively reacting with hydroxyls since an equal number of hydroxyls containing the ${ }^{16} \mathrm{O}$ and ${ }^{18} \mathrm{O}$ labels would be generated from the reaction of water with the chemisorbed oxygen:

$\mathrm{H}_{2} \mathrm{O}(\mathrm{g})+\mathrm{O}(\mathrm{a}) \rightarrow 2 \mathrm{OH}(\mathrm{a})$

Costello et al. [18] agree with the proposal of a significant role for hydroxyls in $\mathrm{CO}$ oxidation. They demonstrated a significant kinetic isotope effect in the oxidation of $\mathrm{CO}$ in the presence of $\mathrm{H}_{2}$ but not with water, attributing the difference to the different kinetic isotope effects expected for the regeneration of $\mathrm{Au} / \mathrm{Al}_{2} \mathrm{O}_{3}$ sites by hydrogen and water. They speculate that the reaction mechanism involves a bicarbonate intermediate, as

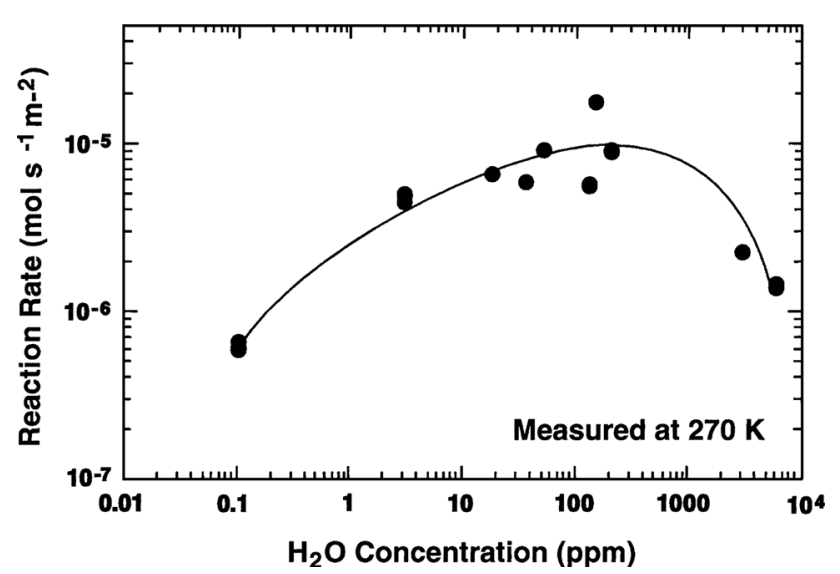

Fig. 1 Dependence of the CO oxidation reaction rate at $270 \mathrm{~K}$ over $50 \mathrm{mg}$ of $1 \mathrm{wt} \% \mathrm{Au} / \mathrm{TiO}_{2}$ on the moisture concentration in the reactant gas (measured at the inlet). Reproduced from Ref. [14] with permission from Elsevier proposed by Thompson, and illustrated in Fig. 2. Deactivation of the catalyst occurs through the dehydration of the bicarbonate to form an unreactive carbonate. Reversing the deactivation step (2) involves nucleophilic attack of the water on the Au-carbonate bond which they expect to show a weak kinetic isotope effect whereas the equivalent hydrogen reaction (3) would exhibit a much stronger effect. This view of the reaction mechanism was echoed by Fujitani et al. in a recent perspective article [19].

$\mathrm{Au}-\mathrm{CO}_{3}-\mathrm{Al}+\mathrm{H}_{2} \mathrm{O} \rightarrow \mathrm{Au}-\mathrm{CO}_{3} \mathrm{H}+\mathrm{Al}-\mathrm{OH}$

$\mathrm{Au}-\mathrm{CO}_{3}-\mathrm{Al}+\mathrm{H}_{2} \rightarrow \mathrm{Au}-\mathrm{CO}_{2} \mathrm{H}+\mathrm{Al}-\mathrm{OH}$

Davis and Ide recently reviewed [20] evidence for the involvement of hydroxyls in gold catalysis of $\mathrm{CO}$ and alcohol oxidation drawing analogies with results from electrochemical studies. They strongly support the view that hydroxyls are critical intermediates in both reactions and propose that at high $\mathrm{pH}$ the $\mathrm{OH}^{-}$present in solution makes the role of the gold/oxide interface less important.

Despite the strong support for the important role of the hydroxide on gold, Okumura [21] advances an alternative to the views of Haruta and Bond and Thompson suggesting that the role of water is to stabilise $\mathrm{O}^{2-}, \mathrm{O}_{2}^{2-}$ and other oxygen states through hydrogen bonding at the surface thereby providing a higher concentration of reactants to trap the CO. However, their hybrid DFT calculations do not explicitly consider the $\mathrm{CO}+\mathrm{OH}$ reaction pathway which other authors regard as the lowest energy pathway. Chandler and coworkers [22] express similar views to Okumura in their study of $\mathrm{Au} / \mathrm{TiO}_{2}$ surfaces with FTIR and isotope labelled water. They argue that there is no direct involvement of $\mathrm{OH}$ groups from the $\mathrm{TiO}_{2}$ in the reaction mechanism, nor do they support the transfer of $\mathrm{OH}$ from $\mathrm{TiO}_{2}$ to the gold. Instead, they suggest that the role of the

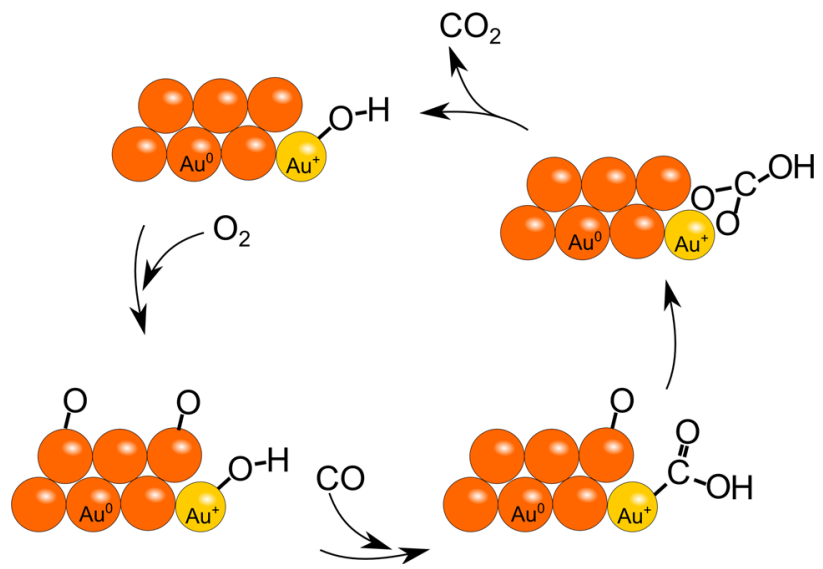

Fig. 2 The bicarbonate model for the role of hydroxyl groups in the oxidation of carbon monoxide at gold surfaces. Adapted with permission from Costello's model from Ref. [18] 
hydroxyl groups on the support is to stabilise water near the $\mathrm{Au} / \mathrm{TiO}_{2}$ interface and it is proton transfer reactions involving water that are crucial with a $* \mathrm{OOH}$ intermediate forming on the gold surface from $\mathrm{O}_{2}$ that reacts readily with $\mathrm{CO}$ to form ${ }^{*} \mathrm{COOH}$ and $\mathrm{O} *$. Decomposition of the $* \mathrm{COOH}$ intermediate is again facilitated by the presence of water.

\subsection{Alcohol Oxidation \& Selectivity of $\mathrm{OH}$ Against $O$ on Gold Surfaces}

Whilst there is considerable support for the involvement of hydroxyls in the oxidation of $\mathrm{CO}$, other reactions such as the oxidation of alcohols at gold surfaces are less likely to be attributed to hydroxyls, Camellone [23] for example considers only preadsorbed molecular oxygen in the oxidation of alcohols. Furthermore, recent DFT calculations by Mullen et al. show that the oxidation of allyl alcohols by hydroxyls on $\mathrm{Au}(111)$ has a higher activation energy than by $\mathrm{O}$ (a) [24]. Later Mullen's calculations were extended [25] to suggest that $\mathrm{OH}$ does accelerate the selective oxidation of the allyl alcohol, and further that $\mathrm{O}(\mathrm{a})$ also catalyses the combustion reaction. In their view, the presence of water is important to maintain the hydroxyl concentration and drive the reaction towards the desired selective oxidation.

Echoing Chandler's views, $\mathrm{Hu}$ and coworkers have recently used a combined DFT molecular dynamics method to study [26] the effect of a water solvent on oxidation reactions over gold surfaces and found that water facilitates oxygen activation to form $\mathrm{OOH}^{*}$. In fact, $\mathrm{Hu}$ and coworkers conclude that hydrogen transfer to the dioxygen in this system is always via a water molecule rather than a hydroxide. They suggest that the role of the water is multifaceted including electron donation to the reactant and stabilizing transition states, echoing Robert's comments [4] on the importance of short lived states in overall reaction mechanisms.

\subsection{Other Transition Metal Surfaces}

As the early work of Boswell and Beeck demonstrated, water is integral to catalytic processes on metal surfaces other than gold, with hydroxyl groups frequently proposed as key intermediates. Bergeld et al. for example provide evidence [27] for a similar mechanism for $\mathrm{CO}$ oxidation occurring on $\mathrm{Pt}(111)$ as was discussed above for gold. In a TPD study of $\mathrm{CO} / \mathrm{O}_{2}$ on $\mathrm{Pt}(111)$ they show the introduction of small amounts of water leads to an oxidation pathway at $200 \mathrm{~K}$, approximately $100 \mathrm{~K}$ lower than in the absence of water, and demonstrate that it is hydroxyls at the surface that provide this low temperature pathway. Another example on platinum is Besson's 2011 [28] study of the oxidation of higher alcohols over platinum/carbon catalysts. Non-polar solvents are often used in the oxidation of long chain alcohols because of their poor solubility in water; Besson and coworkers reported that water added to a dioxane solvent increased the rate of reaction over a $\mathrm{C} / \mathrm{Pt}$ catalyst. They attributed this behaviour to an indirect effect of the solvent changing the affinity of the substrate and the product for the catalyst surface or the solvent. The basic nature of water also assists $\mathrm{H}$-abstraction from the alcohol which is the first step of the reaction during the dissociative chemisorption of the alcohol molecule on the catalyst surface. Subsequently, in a combined experimental/DFT study [29] Besson and coworkers changed their view slightly suggesting that the rate enhancement in the presence of water is not due to the water itself but, as in the case of $\mathrm{CO}$ oxidation, to hydroxyl groups formed by reaction of water with dissociated oxygen at the surface of the catalyst, Fig. 3.

On $\mathrm{Pd} / \mathrm{TiO}_{2}$ catalysts Leung and coworkers reported [30] that water accelerates the oxidation of formaldehyde. By comparing activities between good quality and defective $\mathrm{TiO}_{2}$ surfaces they identify the activation of oxygen on the $\mathrm{TiO}_{2}$ and its transfer to the reactive site at the $\mathrm{TiO}_{2} / \mathrm{Pd}$ interface as the key factor determining the catalyst's activity. They suggest that the role of the water is to facilitate both these steps through the formation of hydroxyls at defective sites on the $\mathrm{TiO}_{2}$ which then aid the diffusion of active oxygen to the reactive site where a concerted reaction occurs between the activated oxygen, the formaldehyde and two hydroxyls. Some support for elements of this mechanism comes from a study by $\mathrm{Hu}$ on the role of water on $\mathrm{CO}$ dissociation on $\mathrm{TiO}_{2}$ [31]. The results show that water dissociates readily into $\mathrm{OH}$ groups and these facilitate $\mathrm{O}_{2}$ adsorption. Furthermore, they show that the effect of the $\mathrm{OH}$ group on $\mathrm{O}_{2}$ adsorption is surprisingly long-range.

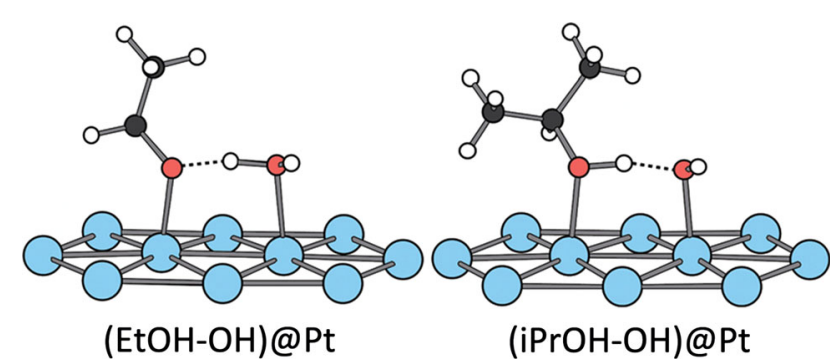

Fig. 3 Chibani et al's calculations show hydroxyl's adsorbed in an a-top position; ethanol interacts with a neighbouring platinum and its hydroxyl hydrogen is transferred to the pre-adsorbed hydroxyl group along the adsorption path, leading to the $\mathrm{H}_{2} \mathrm{O}-\mathrm{EtO}$ structure shown above. Reproduced from Ref. [29] with permission from The Royal Society of Chemistry 
The practical implications of the involvement of water in catalytic reactions over palladium are emphasised by a recent elegant study by Caporali et al. [32] on the direct oxidation of carbon monoxide and hydrocarbons (modelled by propene) in the after treatment of diesel exhaust. Using isotopically labelled oxygen in their feedstock, the study demonstrated that the source of the oxygen in the oxidation of CO is water (Fig. 4). This suggests that the oxygen plays a secondary role, perhaps activating the water which can then react with the $\mathrm{CO}$. In other words the action of the water is to facilitate the transfer of oxygen faster. It is interesting that dioxygen extracting hydrogen from the water is not contributing to the reaction pathway towards $\mathrm{CO}_{2}$, suggesting either unique properties for the hydroxyl group containing the oxygen atom from the water (the authors suggest a radical species) or perhaps a direct $\mathrm{CO}$ water interaction before dissociation.

\subsection{Fischer-Tropsch Catalysis}

On cobalt based Fischer-Tropsch catalysts, water has a profound influence with an increased water partial pressure resulting in a significantly increased surface specific activity as well as increased chain growth, lower methane and higher $\mathrm{C}^{5+}$ selectivity [33]. These phenomena depend on the identity of the support and lead to support effects on turnover rates at low CO conversions [34] but the origin of the effects remains a puzzle.

On $\mathrm{TiO}_{2}$ based Co catalysts Iglesias [34] suggests the water could be reversing the decoration effects observed
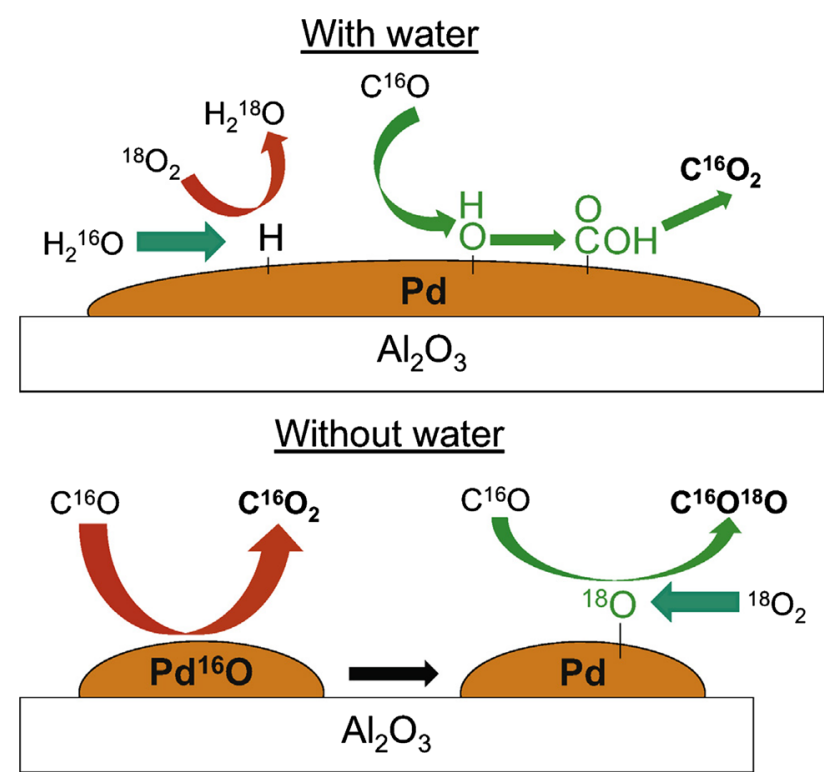

Fig. 4 Schematic illustration of the predominant reaction pathways for $\mathrm{CO}$ oxidation with and without $\mathrm{H}_{2} \mathrm{O}$. Reproduced from Ref. [32] when the catalyst is reduced and which serve to block Co sites. However, such decoration is not seen with either silica supported catalysts nor on cobalt powder and yet both still show an enhancement with the presence of water. Iglesias suggests [34] that in small-pore supports, such as $\mathrm{Al}_{2} \mathrm{O}_{3}$ and $\mathrm{SiO}_{2}, \mathrm{CO}$ and $\mathrm{H}_{2}$ transport within the porous structure is facilitated by an intra-pellet water phase, increasing the accessibility of isolated regions within the pellets.

A combined micro-reactor and computational study of ruthenium catalysts by Neurock and coworkers [35] considered the influence of water on two different pathways leading to a common $\mathrm{HCOH}$ intermediate in FischerTropsch synthesis: the first involving hydrogenation of $\mathrm{CO}$ via the oxygen ("formyl" route) and the other via the carbon ("hydroxymethylidyne" route). They point out that their experimental data is insufficient to discriminate between the likely hydrogenation intermediates, but DFT calculations of the free energies of the of $\mathrm{H}, \mathrm{OH}$ and $\mathrm{H}_{2} \mathrm{O}$ intermediates suggest that $\mathrm{H}_{2} \mathrm{O}$ has the highest surface concentration under reaction conditions. Whilst the DFT calculations suggest water would have a minimal overall impact on the formyl route, protonation of the hydroxymethylidyne intermediate via a $\mathrm{H}_{3} \mathrm{O}^{+}$intermediate decreases the activation barrier significantly and was consistent with the experimentally observed rate enhancements when water was co-fed into the reaction. Similarities between the ruthenium and cobalt catalysts suggest that similar conclusions are valid for the reaction over cobalt catalysts.

Michel et al. [36], discussing the hydrogenation of ketones, and the conversion of levulinic acid into gammavalerolactone in particular, suggest that the promotion of catalytic reactions by water is a common theme for metals that have relatively high d-band energies; this includes $\mathrm{Ru}$, $\mathrm{Co}$ and $\mathrm{Ni}$. Pd and $\mathrm{Pt}$ on the other hand are insensitive to the presence of water. In agreement with Neurock et al. Michel et al.'s DFT calculations suggest a protonated water molecule is the key intermediate that promotes a reaction pathway involving an alkoxy intermediate whereas in the absence of water the hydrogenation pathway involves an alkyl intermediate.

\subsection{The Indirect Interactions of Water in Heterogeneous Catalysis}

In the discussion above, a couple of examples suggest that one of the roles of water is to provide a means of hydrogen transfer between active states and there are also suggestions that in some cases water acts by stabilising reaction intermediates. Roberts [4] drew attention to Marcus' model [37] explaining Sharpless' observation [38] of rate enhancements in oil/water suspensions and two further 
examples will serve to illustrate the relevance of such phenomena. The first is in a similar vein to the Marcus' model; Qiu and coworkers studied [39] the selective oxidation of benzyl alcohol to benzaldehyde over Ru/CNTs in an oil/water emulsion. They reported significant enhancements in rate with the presence of water which they attribute to a more rapid oxidation of the alcohol in the water phase and a rapid extraction of the product back into the non-polar solvent, Fig. 5. The second case that should be mentioned is that of heteropolyacids. Micek-Ilnicka's review [40] highlights the multifaceted role of water which can be present in HPA as protonated or non protonated monomers and also as clusters but of particular interest in this section is the influence of water on the secondary structure of the HPA indirectly controlling the reaction.

\section{Future Work}

Roberts' final paper [4] argues the case for surface science to pursue a different type of experiment than the classic sequential adsorption protocol if it is to provide useful information on the role of water in catalysis. This continued a theme developed over the preceding two decades [41] that emphasised the importance of "dynamic" experiments in surface science where the coadsorption of reactants allows the interception of very short lived species by a second reactant. This opens up reaction pathways which would not be available in experiments in which reactants are dosed sequentially. An excellent early example of the approach is a study [42] of $\mathrm{CO}$ oxidation at aluminium surfaces, which occurs readily at $80 \mathrm{~K}$ when oxygen and carbon monoxide are dosed simultaneously, giving rise to surface carbonate and its decomposition products, Fig. 6 . On the other hand, carbon monoxide neither reacts nor physisorbs with clean or pre-oxidised

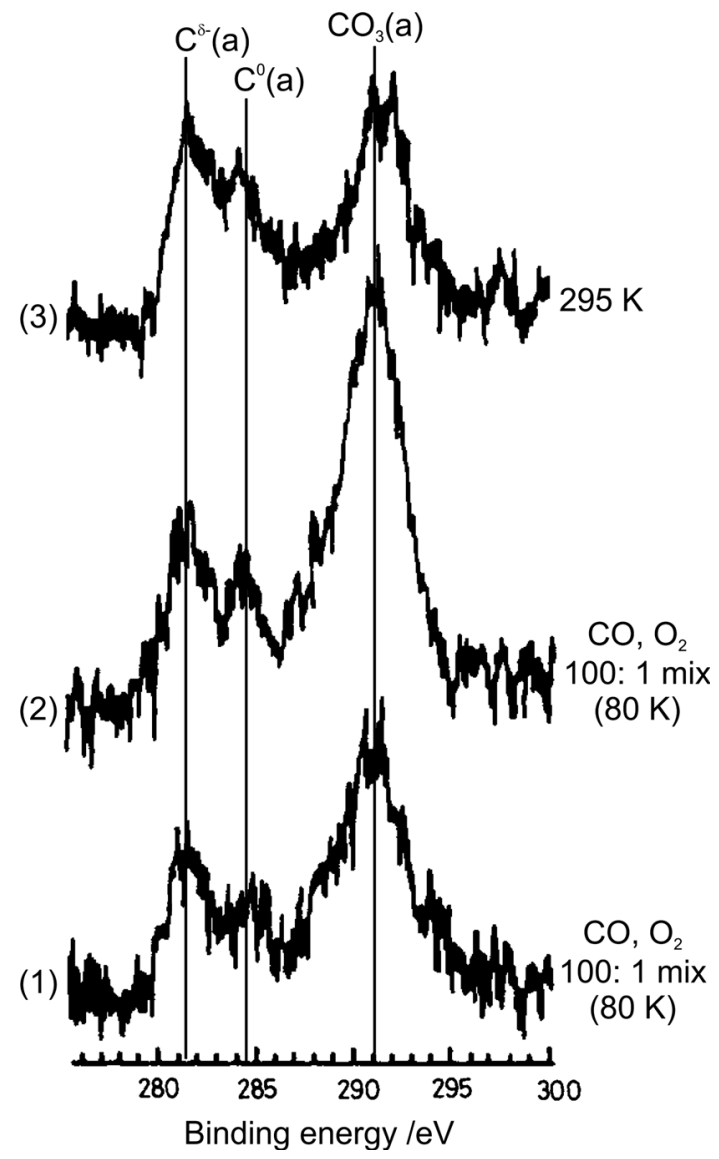

Fig. $6 \mathrm{C}(1 \mathrm{~s})$ spectra showing the oxidation of $\mathrm{CO}$ at an aluminium surface in a coadsorption experiment carried out at $80 \mathrm{~K}$. (1) After a $\mathrm{CO}: \mathrm{O}_{2}$ 100:1 mix was dosed to the aluminium surface at $3 \times 10^{-6}$ torr for $5 \mathrm{~min}$; (2) further dose of $1 \times 10^{-5}$ torr for $5 \mathrm{~min}$; (3) After warming to $295 \mathrm{~K}$. Adapted from Ref. [42] with permission from The Royal Society of Chemistry

aluminium surfaces at $80 \mathrm{~K}$. This experiment reported [42] in 1987 nicely demonstrates that the short lived oxygen states theorized to be present between the adsorption of
Fig. 5 Scheme 1. Schematic model of multiphase reaction system for the selective oxidation of benzyl alcohol to benzaldehyde over Ru/CNTs. Reproduced with permission from Ref. [39]

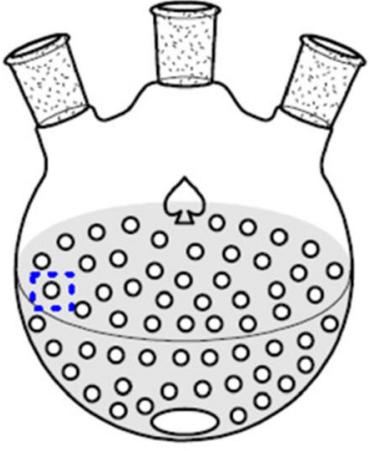

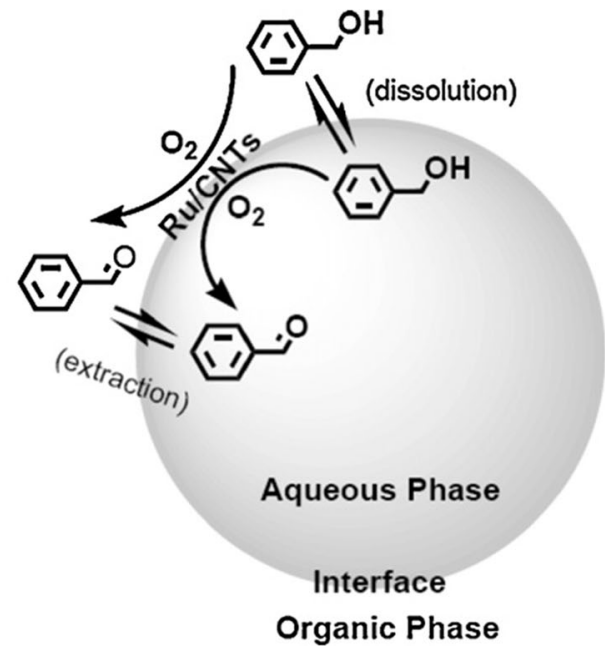


molecular oxygen at an aluminium surface and the formation of the chemisorbed state, Fig. 7, are highly reactive. Further direct evidence for such states was published by Ertl and coworkers [43].

A similar approach led to a subtly different phenomenon for the oxidation of ammonia at zinc surfaces. Roberts chose the zinc surface because of its very low reactivity, oxygen having a sticking probability of $<10^{-3}$ on this surface. At low temperatures coadsorbed ammonia leads to oxygen reaction at a rate $10^{3}$ times faster than the clean surface, Fig. 8 [44]. This implied direct interaction between the ammonia and the molecular oxygen state at the surface with the reaction kinetics indicating the involvement of a precursor complex between the ammonia and the dioxygen. Based on the known pyridine-dioxygen complex this was suggested to involve ammonia acting as a Lewis base towards the oxygen.

Roberts speculated that the same model can be used to explain the high reactivity of water to coadsorbed dioxygen, on silver and zinc surfaces $[45,46]$ where the presence of water increases the rate of oxygen dissociation dramatically. However, IR spectra and computational studies suggest that the $\mathrm{H}_{2} \mathrm{O}-\mathrm{O}_{2}$ complex is hydrogen bonded [47] (Fig. 9) rather than $\mathrm{T}$ shaped as in the case of ammonia dioxygen. Never-the-less the water-dioxygen interaction leads to very high activities towards the oxidation of water and the formation of high concentrations of hydroxyl species at metal surfaces; with the correct choice of conditions, almost pure hydroxyl adlayers can be generated in the absence of interfering oxygen states such as chemisorbed oxygen or water. Figure 10 for example shows XPS and HREELS spectra of a near monolayer of hydroxyl groups at a $\mathrm{Cu}(111)$ surface [46].

Roberts' argued [4] that using such dynamic surface science methods is necessary to gain information on relevant intermediates in catalytic reactions, and also to explore possible reaction pathways that "static" surface science experiments would not detect. The validity of this approach has already been demonstrated with the imide

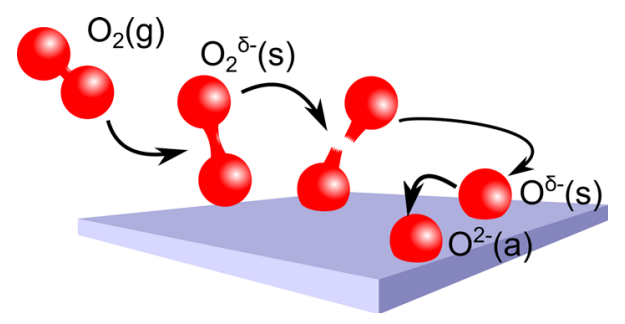

Fig. 7 Schematic of the short lived species that exist transiently during the chemisorption of dioxygen at a metal surface. These include a molecular state and a transient atomic oxygen state that precedes the unreactive chemisorbed state

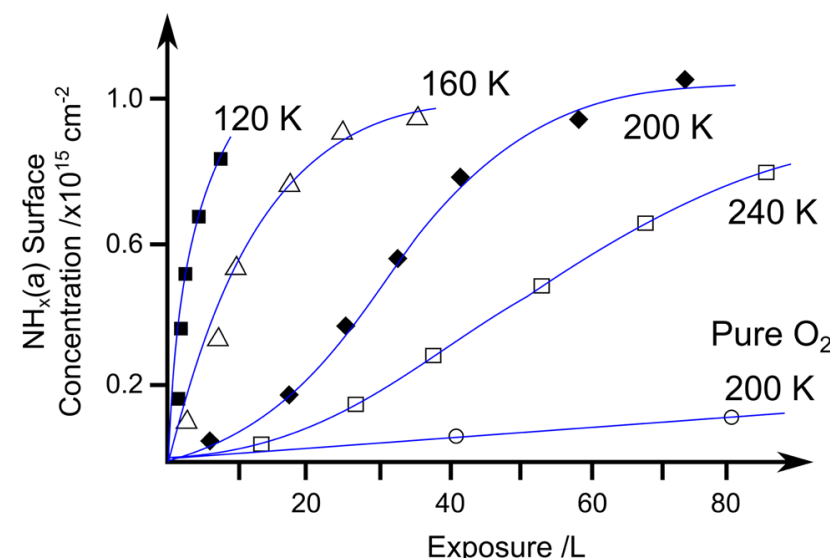

Fig. 8 Surface concentration of $\mathrm{NH}_{\mathrm{x}}$ at a $\mathrm{Zn}(\mathrm{OOO} 1)$ surface exposed to an ammonia-dioxygen $(2: 1)$ mixture at different temperatures. The strong negative temperature dependence of the initial rate is evidence for the involvement of a surface $\mathrm{O}_{2}-\mathrm{NH}_{3}$ complex. The 'catalytic' role of the ammonia in dioxygen bond cleavage is highlighted by comparison with pure dioxygen at $200 \mathrm{~K}$. Adapted from Ref. [44] with permission from the Royal Society of Chemistry

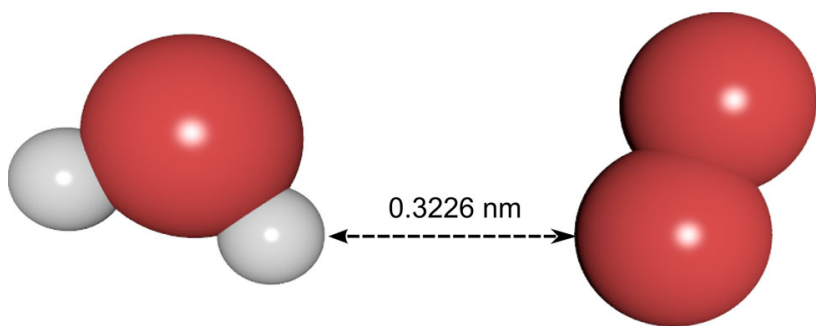

Fig. 9 Geometric arrangement of water and dioxygen complex drawn from the dimensions calculated in Ref. [47]

species on $\mathrm{Cu}(110)[48,49]$ but has yet to be exploited in the case of the hydroxyls.

\section{Summary}

A sophisticated understanding of the reaction pathways followed by water at surfaces has developed over the last 80 years to answer Beeck's challenge of understanding the role of water in heterogeneous catalysis. Computational studies have contributed greatly to this increased understanding by elucidating the unexpected complexity in the behaviour of water. We now suspect that water may play a role as a feedstock to create hydroxyls, as a proton transfer agent through $\mathrm{H}_{3} \mathrm{O}^{+}$and even, as Roberts highlighted, simply as a means of stabilising reactive intermediates through hydrogen bonding. Roberts suggested that there remains a great deal of work to be done in exploring the role of water in heterogeneous catalysis and this short review confirms that opinion highlighting a number of cases where definitive experimental data is still required. 


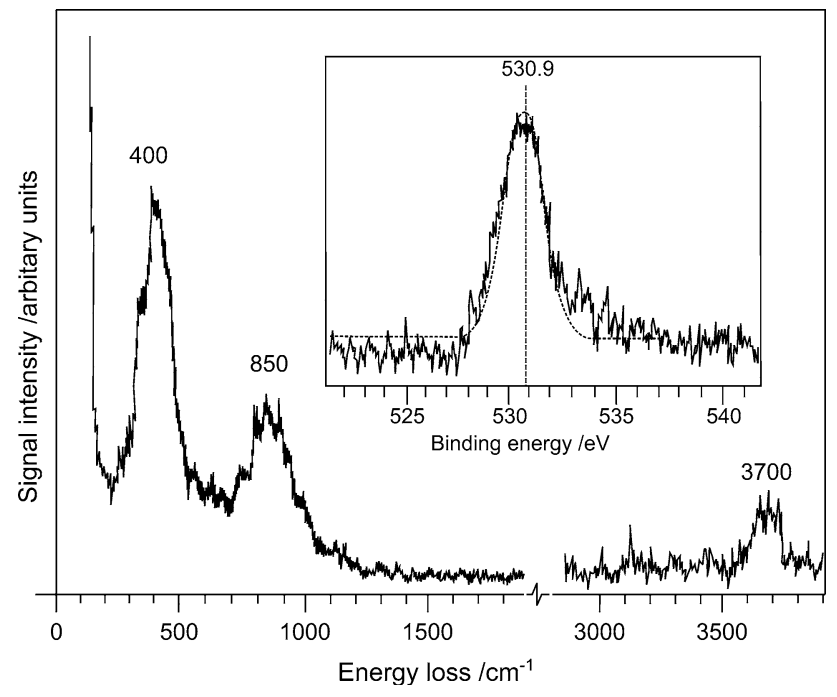

Fig. 10 HREELs and XP spectra of a high coverage of hydroxyls $\left(4.7 \times 10^{14} \mathrm{~cm}^{-2}\right)$ synthesised at a $\mathrm{Cu}(111)$ surface by the coadsorption of 36 Langmuir of a $52: 1 \mathrm{H}_{2} \mathrm{O}: \mathrm{O}_{2}$ mix at $160 \mathrm{~K}$ followed by warming to $190 \mathrm{~K}$. Figure redrawn with permission from Ref. [46], further details are available in the original paper

Acknowledgments The author's research has been supported by EPSRC, currently through Grant EP/I038748/1. The author would also like to express his gratitude to Professor M. W. Roberts for the support and inspiration he has provided during the authors career.

Open Access This article is distributed under the terms of the Creative Commons Attribution 4.0 International License (http://crea tivecommons.org/licenses/by/4.0/), which permits unrestricted use, distribution, and reproduction in any medium, provided you give appropriate credit to the original author(s) and the source, provide a link to the Creative Commons license, and indicate if changes were made.

\section{References}

1. Carr C, Ho S, Lewis D, Owen E, Roberts M (1985) J Text Inst 76:419-424

2. Freund HJ, Roberts MW (1996) Surf Sci Rep 25:225-273

3. Roberts MW (2000) Catal Lett 67:1-71

4. Roberts MW (2014) Catal Lett 144:767-776

5. Boswell MC (1922) Trans R Soc Can 16:1

6. Boswell MC (1922) Trans R Soc Can 17:1

7. Beeck O (1935) Nature 136:1028-1029

8. Hodgson A, Haq S (2009) Surf Sci Rep 64:381-451

9. Carrasco J, Hodgson A, Michaelides A (2012) Nat Mater 11:667-674

10. Burgess R, Buono C, Davies PR, Davies RJ, Legge T, Lai A, Lewis R, Morgan DJ, Robinson N, Willock DJ (2015) J Catal 323:10-18

11. Sterrer M, Freund H-J (2013) Catal Lett 143:375-385

12. Thiel PA, Madey TE (1987) Surf Sci Rep 7:211-385

13. Henderson MA (2002) Surf Sci Rep 46:1-308

14. Daté M, Haruta M (2001) J Catal 201:221-224
15. Cunningham DAH, Vogel W, Haruta M (1999) Catal Lett 63:43-47

16. Bond GC, Thompson DT (2000) Gold Bull 33:41-50

17. Ojifinni RA, Froemming NS, Gong J, Pan M, Kim TS, White JM, Henkelman G, Mullins CB (2008) J Am Chem Soc 130:6801-6812

18. Costello CK, Yang JH, Law HY, Wang Y, Lin J-N, Marks LD, Kung MC, Kung HH (2003) Appl Catal General 243:15-24

19. Fujitani T, Nakamura I, Haruta M (2014) Catal Lett 144:1475-1486

20. Ide MS, Davis RJ (2014) Acc Chem Res 47:825-833

21. Okumura M, Kitagawa Y, Haruta M, Yamaguchi K (2005) Appl Catal General 291:37-44

22. Saavedra J, Doan HA, Pursell CJ, Grabow LC, Chandler BD (2014) Science 345:1599-1602

23. Camellone MF, Marx D (2014) J Phys Chem C 118:20989-21000

24. Mullen GM, Zhang L, Evans EJ, Yan T, Henkelman G, Mullins CB (2014) J Am Chem Soc 136:6489-6498

25. Mullen GM, Zhang L, Evans EJ, Yan T, Henkelman G, Mullins CB (2015) Phys Chem Chem Phys 17:4730-4738

26. Liu J, Cao X-M, Hu P (2014) Phys Chem Chem Phys $16: 4176-4185$

27. Bergeld J, Kasemo B, Chakarov DV (2001) Surf Sci 495:L815L820

28. Frassoldati A, Pinel C, Besson M (2011) Catal Today 173:81-88

29. Chibani S, Michel C, Delbecq F, Pinel C, Besson M (2013) Catal Sci Technol 3:339-350

30. Huang H, Ye X, Huang H, Zhang L, Leung DYC (2013) Chem Eng J 230:73-79

31. Liu LM, McAllister B, Ye HQ, Hu P (2006) J Am Chem Soc 128:4017-4022

32. Caporali R, Chansai S, Burch R, Delgado JJ, Goguet A, Hardacre C, Mantarosie L, Thompsett D (2014) Appl Catal B Environ 147:764-769

33. Fischer N, Clapham B, Feltes T, Claeys M (2015) ACS Catal 5:113-121

34. Iglesia E (1997) Appl Catal General 161:59-78

35. Hibbitts DD, Loveless BT, Neurock M, Iglesia E (2013) Angew Chem Int Ed 52:12273-12278

36. Michel C, Zaffran J, Ruppert AM, Matras-Michalska J, Jędrzejczyk M, Grams J, Sautet P (2014) Chem Commun 50:12450-12453

37. Jung Y, Marcus RA (2007) J Am Chem Soc 129:5492-5502

38. Narayan S, Muldoon J, Finn MG, Fokin VV, Kolb HC, Sharpless KB (2005) Angew Chem Int Ed 44:3275-3279

39. Yang X, Wang X, Qiu J (2010) Appl Catal General 382:131-137

40. Micek-Ilnicka A (2009) J Mol Catal Chem 308:1-14

41. Carley AF, Davies PR, Roberts MW (2011) Catal Today 169:118-124

42. Carley A, Roberts M (1987) J Chem Soc Chem Commun 5:355-356

43. Brune H, Wintterlin J, Behm RJ, Ertl G (1992) Phys Rev Lett 68:624

44. Carley A, Yan S, Roberts M (1990) J Chem Soc Faraday Trans 86:2701-2710

45. Carley A, Davies P, Roberts M, Thomas K (1990) Surf Sci 238:L467-L472

46. Carley A, Davies P, Roberts M, Shukla N, Song Y, Thomas K (1994) Appl Surf Sci 81:265-272

47. Sennikov PG, Ignatov SK, Schrems O (2005) ChemPhysChem $6: 392-412$

48. Afsin B, Davies P, Pashuski A, Roberts M (1991) Surf Sci 259:L724-L728

49. Woodruff DP (2008) Chem Soc Rev 37:2262-2273 